\title{
Article \\ Tunable Temperature Characteristic of Terahertz Bragg Fiber Filled with Liquid Water
}

\author{
Cuijuan Guo ${ }^{1}$, Mengya Su ${ }^{1}$, Jia Shi ${ }^{1,2, *} \mathbb{C}$, Linlin Tian ${ }^{1}$, Wei Xu ${ }^{1,2} \oplus$, Hongli Chen ${ }^{1}$, Hua Bai ${ }^{1}$, Zhitao Xiao ${ }^{1}$, \\ Degang $\mathrm{Xu}^{2}$ and Jianquan $\mathrm{Yao}^{2}$
}

1 Tianjin Key Laboratory of Optoelectronic Detection Technology and System, School of Electrical and Electronic Engineering, Tiangong University, Tianjin 300387, China; guocuijuan@tiangong.edu.cn (C.G.); 1930071266@tiangong.edu.cn (M.S.); 1930071269@tiangong.edu.cn (L.T.); xuwei@tiangong.edu.cn (W.X.); chenhongli@tiangong.edu.cn (H.C.); baihua@tiangong.edu.cn (H.B.); xiaozhitao@tiangong.edu.cn (Z.X.)

2 Key Laboratory of Opto-Electronic Information Technology, Ministry of Education, School of Precision Instrument and Opto-Electronics Engineering, Tianjin University, Tianjin 300072, China; xudegang@tju.edu.cn (D.X.); jqyao@tju.edu.cn (J.Y.)

* Correspondence: shijia@tiangong.edu.cn; Tel.: +86-152-2278-2770

Citation: Guo, C.; Su, M.; Shi, J.; Tian, L.; Xu, W.; Chen, H.; Bai, H.; Xiao, Z.; Xu, D.; Yao, J. Tunable Temperature Characteristic of Terahertz Bragg Fiber Filled with Liquid Water. Appl. Sci. 2021, 11, 8306. https://doi.org/ 10.3390/app11188306

Academic Editor: Nunzio Cennamo

Received: 6 July 2021

Accepted: 1 September 2021

Published: 7 September 2021

Publisher's Note: MDPI stays neutral with regard to jurisdictional claims in published maps and institutional affiliations.

Copyright: (C) 2021 by the authors. Licensee MDPI, Basel, Switzerland. This article is an open access article distributed under the terms and conditions of the Creative Commons Attribution (CC BY) license (https:// creativecommons.org/licenses/by/ $4.0 /)$.

\begin{abstract}
Hollow-core terahertz (THz) fibers have attracted a lot of research interest due to the low loss and easy inner modification with functional materials. Liquid water has unique properties in the $\mathrm{THz}$ region and has been widely investigated in $\mathrm{THz}$ emission, sensing, and devices. In this paper, a hollow-core THz Bragg fiber with a water defect layer is proposed. The finite element method is used to verify and analyze the tunable temperature characteristic of the water-filled $\mathrm{THz}$ fiber. The numerical analysis results show that the confinement loss and the low-frequency side of the dip near $0.5 \mathrm{THz}$ can be controlled by the temperature of the liquid water. The temperature sensitivity of the $\mathrm{THz}$ fiber is obtained at $0.09614 \mathrm{~dB} \cdot \mathrm{m}^{-1} / \mathrm{K}$ at $0.45 \mathrm{THz}$ with a high core power fraction up to $98 \%$. The proposed $\mathrm{THz}$ fiber has potential applications in $\mathrm{THz}$ interaction with liquid and $\mathrm{THz}$ tunable devices.
\end{abstract}

Keywords: terahertz; liquid water; temperature; tunable devices; hollow-core Bragg fiber

\section{Introduction}

The terahertz $(\mathrm{THz})$ frequency region lies midway between the microwave and the far-infrared wave in the electromagnetic frequency spectrum [1,2], ranging from 0.1 to $10 \mathrm{THz}$. In recent years, the focus on $\mathrm{THz}$ has increased because of its numerous potential applications, including broadband short-distance data transmission, imaging, sensing, defense and security screening, biomedical technology, and spectroscopy [3-10]. However, $\mathrm{THz}$ is still in the development phase because the $\mathrm{THz}$ waveguides are bulky and only propagate in the vacuum, and the $\mathrm{THz}$ sources and detectors remain primitive compared with their optics counterparts in the other frequency region. In order to address these issues, various $\mathrm{THz}$ waveguides have been proposed.

Various THz waveguides have been recently reported, including polymer fibers [11-17], metal tube waveguides [18], metal wire waveguides [19], and so on. The polymer fibers can achieve low loss and good flexibility. The types of $\mathrm{THz}$ fibers include solid-core fibers [16], hollow-core fibers [11-15], and porous-core fibers [17]. Among them, the hollow-core fibers have attracted a lot of research interest due to the low loss and easy inner modification with functional materials. One of its operating principles is based on photonic band gap guidance [20], and they are used in air propagation with low loss owing to the strong confinement of the mode in the core area [21,22]. Another is an antiresonant effect. The hollow-core Bragg fibers, as one of the photonic band gap fibers, provide many advantages including flexible design, easy integration of microfluidic channels directly 
into the fiber cladding, and a wide operation range from far-infrared to $\mathrm{THz}$ compared to the other devices. The fibers, filled with functional materials, supply the chance to enhance the properties of the fibers, attain tunable fibers and related devices, which have been successfully developed in recent years. An argon gas-filled hollow-core fiber was designed as a pulse shaper to generate broadband and tunable time-domain spectroscopy in the $\mathrm{THz}$ by adjusting the argon gas pressure [11]. A fiber filled with methanol $\left(\mathrm{CH}_{3} \mathrm{OH}\right)$ gas in the gas laser was used as the reaction cell to shrink the volume of $\mathrm{THz}$ sources [12]. A THz fiber selectively filled with Potassium Chloride (KCL) was reported as Epsilon-Near-Zero (ENZ) material to ensure near-zero flattened dispersion [13]. The $\alpha$-lactose monohydrate powder was filled in the THz hollow-core Bragg fiber as resonant surface bio-sensor [14]. A $\mathrm{THz}$ hollow-core Bragg fiber with microfluidic channel was demonstrated for non-contact monitoring refractive index (RI) changes in flowing liquids (oil) [15].

Liquid water is one of the most common and easily available materials in nature compared to the aforementioned materials, and it can perform as a very good candidate for $\mathrm{THz}$ devices. Adjacent water molecules form a tetrahedral arrangement of hydrogen bonds. The typical time scales related to the relaxation dynamics of the hydrogen-bonded network of water molecules rotated from or displaced their equilibrium position falls in the picosecond to sub-picosecond [23]. Thereby, liquid water has a strong absorption characteristic in the $\mathrm{THz}$ frequency region. Further, $\mathrm{THz}$ waves, generated from liquid water, have recently been studied [24-26]. In addition, the optical characteristics of liquid water can be affected by the thermal relaxation and resonant process. Both the dipolar orientational and the translational stretch of the hydrogen band could result in absorbance [27]. Therefore, the complex dielectric constant of liquid water could be controlled by temperature. Thus, $\mathrm{THz}$ fibers filled with liquid water turn to be a choice for $\mathrm{THz}$ generation and tunable devices.

In this paper, a hollow-core $\mathrm{THz}$ Bragg fiber with a defect layer in the cladding region is proposed. Liquid water of different temperatures is filled in the defect layer. The temperature characteristics of the proposed $\mathrm{THz}$ fiber are analyzed and verified by the finite element method. The confinement loss and the low-frequency side of the dip near $0.5 \mathrm{THz}$ is temperature controlled. The temperature sensitivity of the $\mathrm{THz}$ fiber is obtained at $0.09614 \mathrm{~dB} \cdot \mathrm{m}^{-1} / \mathrm{K}$ at $0.45 \mathrm{THz}$ with a high core power fraction up to $98 \%$.

\section{Design of the Hollow-Core Bragg Fiber}

The 3D view of the proposed $\mathrm{THz}$ Bragg fiber is shown in Figure 1. The cross section of it is shown in Figure 2. The hollow-core Bragg fiber consists of a hollow core and eleven alternating cyclical layers of photosensitive resin and air with the same thickness. In the cladding region, the layers of photosensitive resin with high RI and air with low RI are radially arranged. The radius of the core is $2.5 \mathrm{~mm}$, and the thickness of layers is $0.5 \mathrm{~mm}$. In the proposed Bragg fiber, the defect layer is introduced by the first layer of air filled with water at different temperatures.

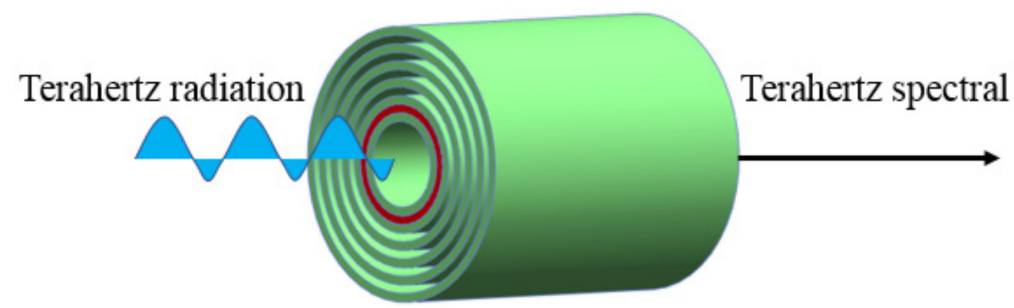

Figure 1. 3D view of the proposed fiber where terahertz $(\mathrm{THz})$ radiation impinges at the center of the fiber. 


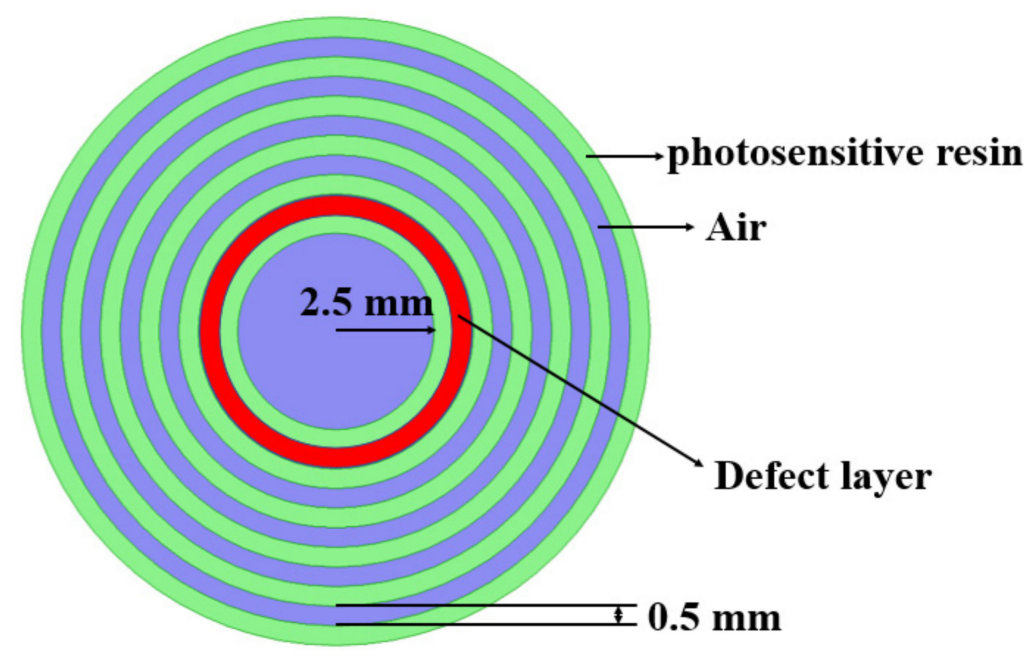

Figure 2. Cross section of the proposed $\mathrm{THz}$ Bragg fiber.

In the Bragg fiber, when the $\mathrm{THz}$ wave propagates from the hollow core to the cladding, the $\mathrm{THz}$ wave is confined in the core due to the Bragg diffraction produced by the periodical cladding. The defect can be considered as a lossy Fabry-Perot cavity. Depending on the phase condition at the core-cladding boundary and the anti-resonant reflection condition in the cladding, the resonant frequency can be expressed by the following equation [28]

$$
f=c /\left(d_{w} \sqrt{n_{w}^{2}-n_{c}^{2}}+d_{p} \sqrt{n_{p}^{2}-n_{c}^{2}}\right)
$$

where $n_{w}$ and $n_{p}$ are RI of the defect layer and photosensitive resin, $d_{w}$ and $d_{p}$ are the corresponding thicknesses, and $n_{c}$ is RI of the core material. In this paper, the core material is air $\left(n_{c}=1\right)$.

Equation (1) shows that the resonant frequency is shifted to the lower frequency with an increase in RI of the defect layer. It indicates that the transmission characteristic can be changed by the RI of the defect layer. Therefore, the transmission characteristic could be controlled through the water with different temperatures filled into the defect layer.

The complex RI of liquid water at different temperatures can be deduced by the dielectric constant. Depending on the double Debye mode, the complex dielectric constant of liquid water can be expressed by [29]

$$
\varepsilon(\omega)=\varepsilon_{\infty}+\frac{\varepsilon_{s}-\varepsilon_{1}}{1+i \omega \tau_{D}}+\frac{\varepsilon_{1}-\varepsilon_{\infty}}{1+i \omega \tau_{1}}
$$

where $\varepsilon_{s}$ is the static dielectric constant at low frequency, $\varepsilon_{\infty}$ is the ultimate dielectric constant at high frequency, $\varepsilon_{1}$ is the transitional dielectric constant between the slow and fast relaxation process, $\tau_{D}$ is the slow relaxation time constant decaying from $\varepsilon_{S}$ to $\varepsilon_{1}$, and $\tau_{1}$ is the fast relaxation time constant decaying from $\varepsilon_{1}$ to $\varepsilon_{\infty}$.

The fundamental description of the complex dielectric constant related to the frequency is [30]

$$
\varepsilon(\omega)=\varepsilon^{\prime}(\omega)-i \varepsilon^{\prime \prime}(\omega)
$$

where $\varepsilon^{\prime}(\omega)$ and $\varepsilon^{\prime \prime}(\omega)$ are the real and imaginary parts of the complex dielectric constant, respectively.

Then, the complex RI can be easily calculated by the following relationships [30]

$$
\hat{n}(\omega)=n(\omega)-i k(\omega)
$$




$$
\begin{gathered}
n(\omega)=\left[\frac{\sqrt{\varepsilon^{\prime}(\omega)^{2}+\varepsilon^{\prime \prime}(\omega)^{2}}+\varepsilon^{\prime}(\omega)}{2}\right]^{1 / 2}, \\
\alpha(\omega)=\frac{4 \pi f}{c}\left[\frac{\sqrt{\varepsilon^{\prime}(\omega)^{2}+\varepsilon^{\prime \prime}(\omega)^{2}}-\varepsilon^{\prime}(\omega)}{2}\right]^{1 / 2}, \\
k(\omega)=\frac{c \alpha(\omega)}{2 \omega}
\end{gathered}
$$

where $n(\omega)$ is the real part of the complex RI, which represents RI, $k(\omega)$ is the imaginary part of the complex RI, which represents extinction coefficient, $\alpha(\omega)$ is the absorption coefficient, which represents absorption loss, $f$ is the frequency, and $c$ is the speed of the light wave in the vacuum. The absorption coefficient and RI of photosensitive resin used in this paper can be expressed as [16].

\section{Simulation and Discussion}

The proposed fiber is designed using COMSOL software, and the finer mesh is used in all regions to obtain more accurate results. The electric field distributions of the fundamental mode with water at $280 \mathrm{~K}$ and without water at $0.45 \mathrm{THz}$ are shown in Figure 3. The electric field of the fundamental mode with water leaks less to the cladding than without water. Meanwhile, the fiber filled with water has most of the power guided in the core region, which leads to a lower loss. Thanks to the strong absorption characteristic of liquid water in the $\mathrm{THz}$ frequency region, this contributes to the stronger transverse resonant coupling between core modes and water defect modes.

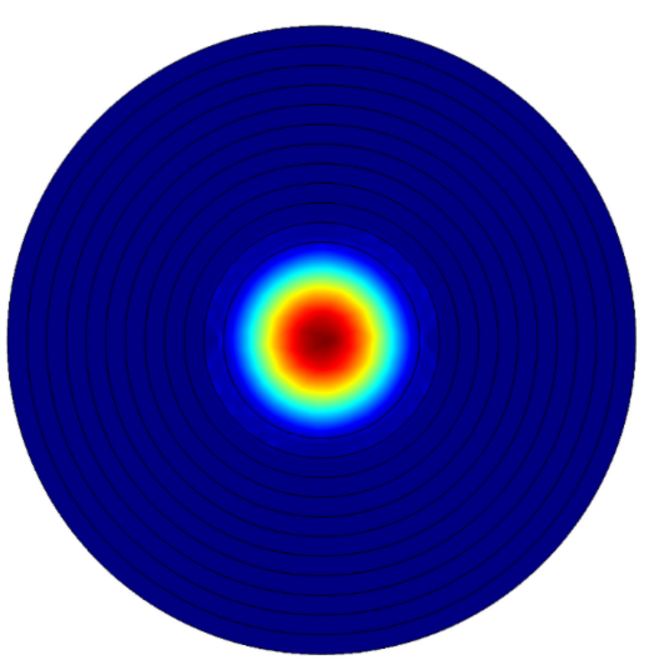

(a)

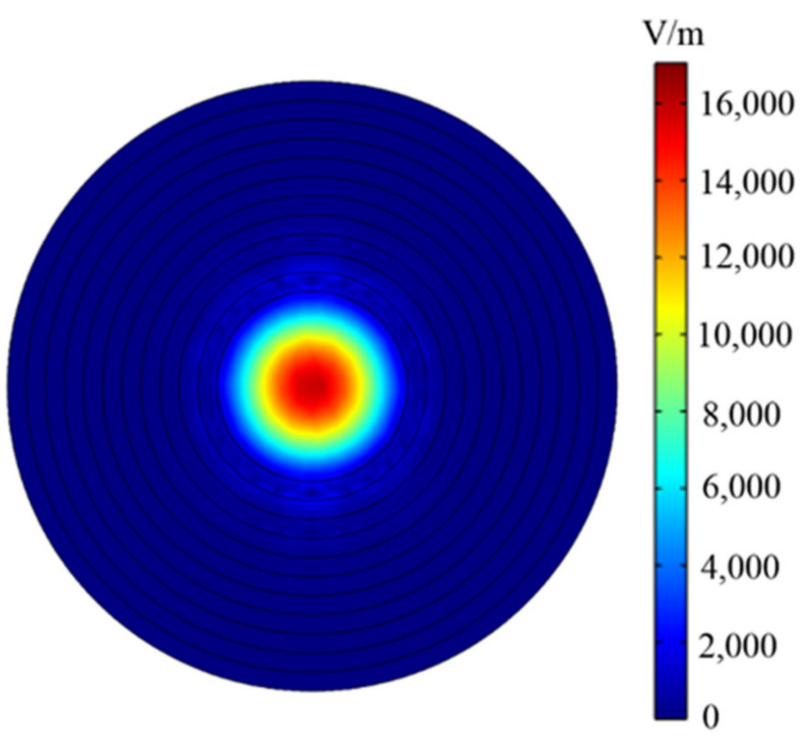

(b)

Figure 3. (a) The electric field distributions of the fundamental mode with water at $280 \mathrm{~K}$ (b) and without water at $0.45 \mathrm{THz}$.

The effective mode area indicates the actual area covered by the propagating $\mathrm{THz}$ wave. The effective mode area of the proposed $\mathrm{THz}$ fiber can be calculated by [31]

$$
A_{e f f}=\frac{\left[\iint|E(x, y)|^{2} d x d y\right]^{2}}{\iint|E(x, y)|^{4} d x d y}
$$


where $E(x, y)$ is the electric field density distribution obtained by the eigenvalue for solving Maxwell's equations. Figure 4 shows the effective mode area with water at different temperatures. The effective mode area takes on two dips at about $0.38 \mathrm{THz}$ and $0.62 \mathrm{THz}$, respectively. Dip1 and the peak of the effective modal area are formed by the transverse resonant coupling between core modes and defect modes. Dip2 is formed by the Bragg resonant. The more leakage of the $\mathrm{THz}$ wave into the cladding region produces a larger effective mode area. With the temperature of water rising from $280 \mathrm{~K}$ to $340 \mathrm{~K}$, the dip 1 of the effective mode area decreases from $280 \mathrm{~K}$ to $340 \mathrm{~K}$; in contrast, the peak from $280 \mathrm{~K}$ to $340 \mathrm{~K}$.

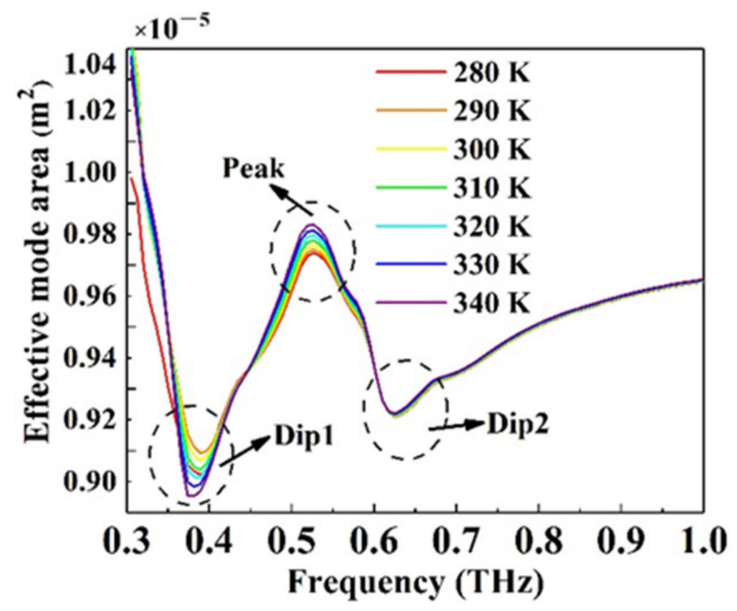

Figure 4. Effective mode area of the fundamental mode with water at different temperatures.

The effective RI of the fundamental mode in the proposed fiber is shown in Figure 5. The real part of the effective RI of the proposed fiber is almost the same, from $280 \mathrm{~K}$ to $340 \mathrm{~K}$. As shown in Figure 5b, in the range of the lower frequency, the imaginary part of effective RI decreases with increased frequency. The inset of Figure $5 b$ shows that, with the frequency range of $0.4 \mathrm{THz}$ to $0.5 \mathrm{THz}$, it displays a temperature effect.

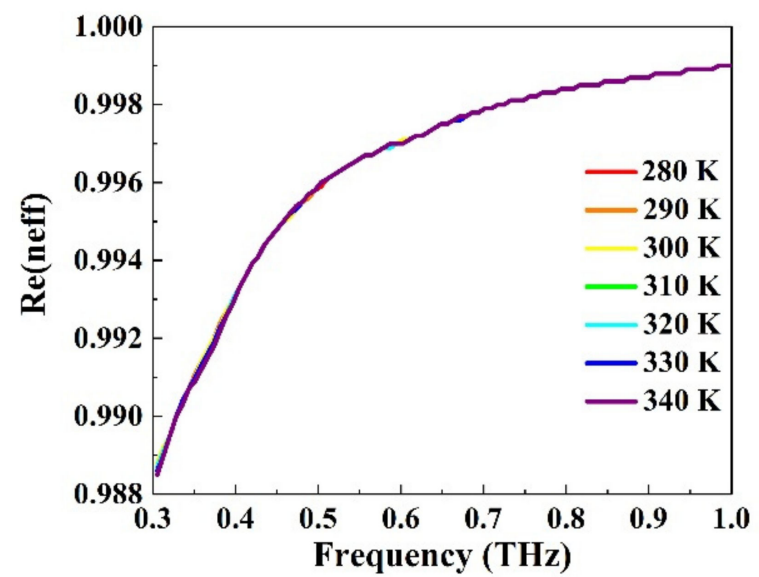

(a)

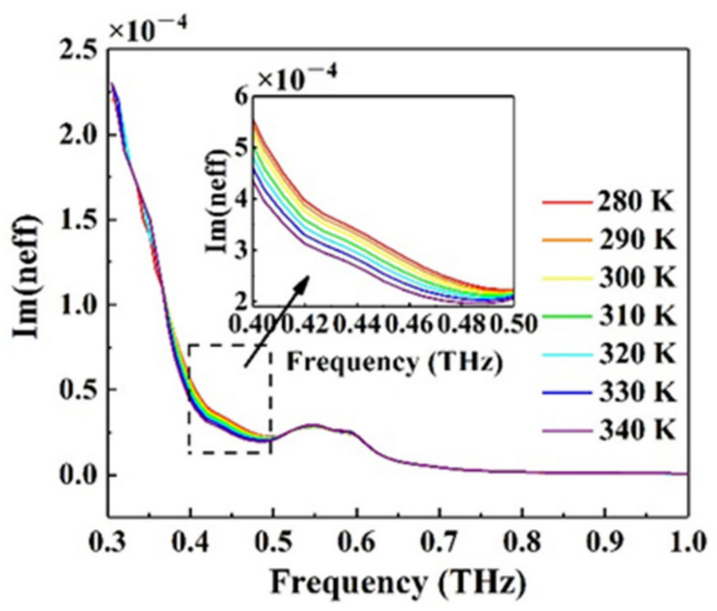

(b)

Figure 5. (a) Real part and (b) imaginary part of the effective refractive index (RI) with water at different temperatures. The inset: detailed information of (b) ranging from $0.4 \mathrm{THz}$ to $0.5 \mathrm{THz}$.

Among few limits in the fiber, the major loss is effective material loss (EML). EML arises from the background material. The hollow core can reduce the amount of the $\mathrm{THz}$ 
wave absorbed by the background material in the core region, which may help reduce the EML. This loss of the proposed PCF can be expressed as [32]

$$
\alpha_{e f f}=\sqrt{\frac{\varepsilon_{0}}{\mu_{0}}}\left(\frac{\int_{\text {mat }} n_{\text {mat }}|E|^{2} \alpha_{\text {mat }} d A}{\left|\int_{\text {all }} S_{z} d A\right|}\right)
$$

where $\varepsilon_{0}$ and $\mu_{0}$ are the permittivity and permeability on the free space, respectively, $n_{\text {mat }}$ is the RI of the photosensitive resin, $\alpha_{m a t}$ is the photosensitive resin absorption loss, $E$ is the modal electric field component, and $S_{z}$ is the $z$ component of the Poynting vector $S_{z}=0.5 \cdot\left(E \times H^{*}\right) \cdot z$ where $H^{*}$ is a complex conjugate of the magnetic field component. Figure 6a indicates that EML is proportional to the operating frequency. It shows that the electric field is more strongly confined within the hollow core at the low-frequency region. Moreover, it has a temperature-insensitive EML.

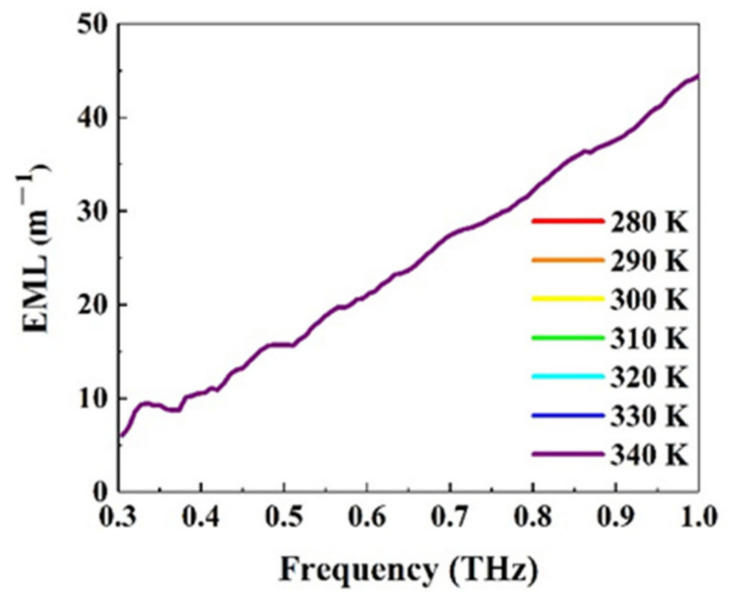

(a)

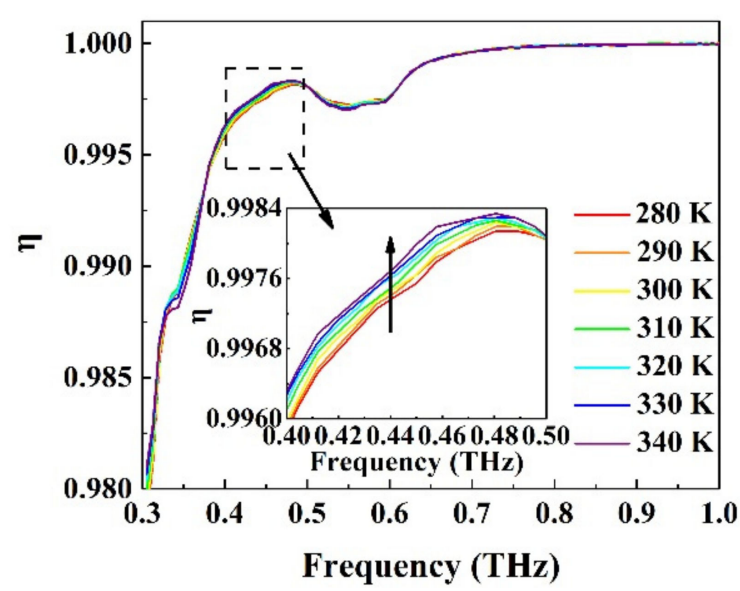

(b)

Figure 6. (a) EML; (b) core power fraction with water at different temperatures. The inset: zoomed-in core power fraction.

The core power fraction indicates the amount of power that propagates through the core region of a fiber. A higher core power fraction is desired in efficient $\mathrm{THz}$ wave transmission application. The core power fraction can be expressed by [33]

$$
\eta=\frac{\int_{i} S_{z} d A}{\int_{\text {all }} S_{z} d A}
$$

where $\eta$ is the mode core power fraction, and the $z$ component of the Poynting vector of the numerator is integrated over the hollow-core region, but the integral range of the denominator is integrated over the entire cross-section region. As shown in Figure $6 \mathrm{~b}$, the core power fraction is greater than $98 \%$. It represents that more than $98 \%$ of power propagates through the core region over the whole range of the frequency. As shown in the inset of Figure $6 b$, the core power fraction increases with the temperature rising within the range of $0.4 \mathrm{THz}$ to $0.5 \mathrm{THz}$ because a stronger interaction is induced between the $\mathrm{THz}$ wave and the liquid water.

The confinement loss indicates how much the $\mathrm{THz}$ wave leaks from the core region to the cladding when the $\mathrm{THz}$ wave propagates through a fiber. The rise of the core and the number of layers lying in the cladding may lead to the leakage of the $\mathrm{THz}$ wave. According to the imaginary of the complex RI, the confinement loss can be quantified using the following expression [6]

$$
\alpha_{C L}=8.686 \times \frac{2 \pi f}{c} \operatorname{Im}\left(n_{e f f}\right)
$$


where $\operatorname{Im}\left(n_{e f f}\right)$ is the imaginary of the effective RI. The confinement loss is shown in Figure 7. It can be observed that a lower confinement loss can be obtained at a frequency of more than $0.4 \mathrm{THz}$. The inset of Figure 7 shows that the frequency range of $0.4 \mathrm{THz}$ to $0.5 \mathrm{THz}$ exhibits an obvious temperature dependence. The $\mathrm{THz}$ wave tends to propagate through materials with a higher RI, and water at high temperatures has a high RI, which causes the lower confinement loss.

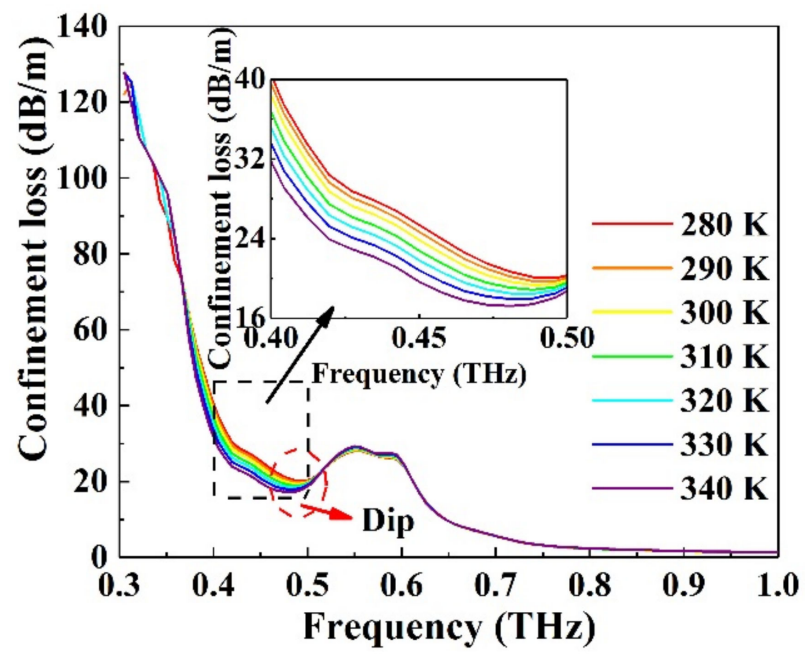

Figure 7. Confinement loss with water for various temperatures. The inset: the detailed information of the confinement loss ranging from $0.4 \mathrm{THz}$ to $0.5 \mathrm{THz}$.

With an increase in the temperature of liquid water, the change trends of the confinement loss at the dip and at $0.45 \mathrm{THz}$ frequency are shown in Figure 8, respectively. The symbols are the simulated data, and the dotted lines are the linear fitting curves. It is apparent that the confinement loss at the dip and at $0.45 \mathrm{THz}$ are both in linear inverse proportion to the temperature. The temperature sensitivity is obtained at $0.09614 \mathrm{~dB} \cdot \mathrm{m}^{-1} / \mathrm{K}$ at $0.45 \mathrm{THz}$, and the coefficient of determination reaches 0.99465 .

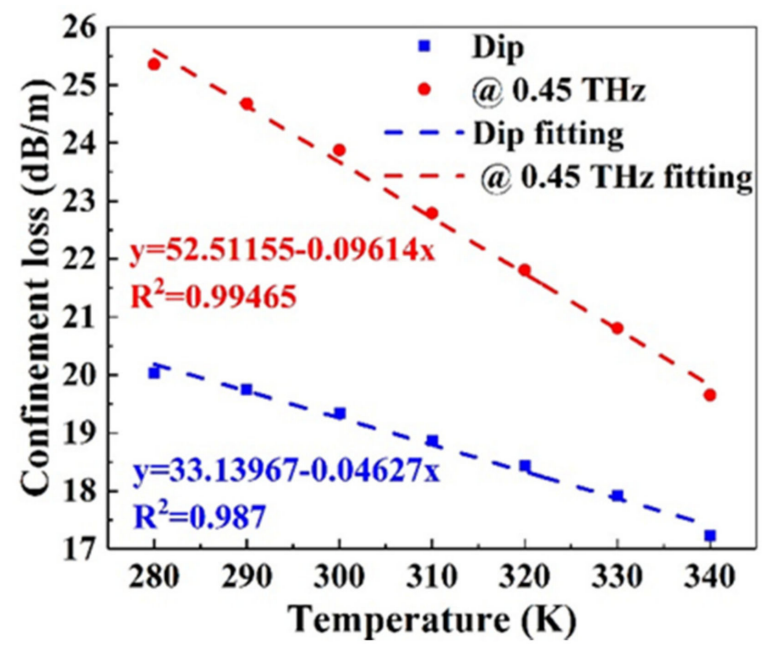

Figure 8. Temperature dependence of the confinement loss at the dip and at $0.45 \mathrm{THz}$.

The proposed $\mathrm{THz}$ fiber can be fabricated by the 3D printing stereolithography (SLA) technique with the advantages of flexible design, fast fabrication, low cost, and convenient applications [14,15]. Liquid water is circulated flowing in the defect layer in the fiber cladding to maintain the same temperature. 


\section{Conclusions}

In conclusion, a hollow-core $\mathrm{THz}$ Bragg fiber with a defect layer is proposed. The tunable temperature characteristic of the liquid water filled in the proposed $\mathrm{THz}$ fiber is analyzed. The results show that the core power fraction is more than $98 \%$ over the frequency range of $0.3 \mathrm{THz}$ to $1 \mathrm{THz}$. The confinement loss and the low-frequency side of the dip near $0.5 \mathrm{THz}$ can be controlled by the temperature of the liquid water. The temperature sensitivity of the proposed $\mathrm{THz}$ fiber is obtained at $0.09614 \mathrm{~dB} \cdot \mathrm{m}^{-1} / \mathrm{K}$ at $0.45 \mathrm{THz}$. With advantages like low loss and cost, controllability, and easy fabrication, the presented fiber has potential applications in $\mathrm{THz}$ tunable devices.

Author Contributions: Conceptualization, J.S. and M.S.; methodology, J.S. and M.S.; software, M.S.; validation, M.S., J.S. and L.T.; data curation, M.S.; writing —original draft preparation, M.S.; writingreview and editing, M.S. and J.S.; supervision, D.X., H.C. and J.Y.; project administration, W.X., H.B. and Z.X.; funding acquisition, J.S. and C.G. All authors have read and agreed to the published version of the manuscript.

Funding: This research was funded by National Natural Science Foundation of China, grant numbers 61905177, 61705164, and 81802118; Natural Science Foundation of Tianjin City, grant numbers 19JCQNJC01400 and 19JCQNJC01600; Education Commission Research Project of Tianjin, grant number 2019KJ015; the key Technologies R\&D Program of Tianjin, grant number 18YFZNGX00040; Open Project Funding of Key Laboratory of Medical Protection for Electromagnetic Radiation, Ministry of Education of China, grant number 2018DCKF002; and the China Postdoctoral Science Foundation funded project, grant numbers 2019M651035 and 2019M661013.

Institutional Review Board Statement: Not applicable.

Informed Consent Statement: Informed consent was obtained from all subjects involved in the study.

Data Availability Statement: The available data has been stated in the article.

Acknowledgments: This manuscript has not been published yet and is not even under consideration for publication elsewhere.

Conflicts of Interest: The authors declare no conflict of interest.

\section{References}

1. Abbott, D.; Zhang, X.C. T-ray imaging, sensing, and retection. Proc. IEEE 2007, 95, 1509-1513. [CrossRef]

2. Tonouchi, M. Cutting-edge terahertz technology. Nat. Photonics 2007, 1, 97-105. [CrossRef]

3. Islam, M.S.; Sultana, J.; Dinovitser, A.; Ng, B.W.; Abbot, D. Zeonex based asymmetrical terahertz photonic crystal fiber for multichannel communication and polarization maintaining applications. Appl. Opt. 2018, 57, 666-672. [CrossRef]

4. Sultana, J.; Islam, M.S.; Faisal, M.; Islam, M.R.; Ng, B.W.; Heidepriem, H.E.; Abbot, D. Highly birefringent elliptical core photonic crystal fiber for terahertz application. Opt. Commun. 2018, 407, 92-96. [CrossRef]

5. Islam, M.S.; Sultana, J.; Dinovitser, A.; Ng, B.W.; Abbot, D. A modified hexagonal photonic crystal fiber for terahertz applications. Opt. Mater. 2018, 79, 336-339. [CrossRef]

6. Habib, M.A.; Anower, M.S. Design and numerical analysis of highly birefringent single mode fiber in THz regime. Opt. Fiber Technol. 2019, 47, 197-203. [CrossRef]

7. Rana, S.; Rakin, A.S.; Hasan, M.R.; Reza, M.S.; Leonhardt, R.; Abbot, D.; Subbaraman, H. Low loss and flat dispersion kagome photonic crystal fiber in the terahertz regime. Opt. Commun. 2018, 410, 452-456. [CrossRef]

8. Mittleman, D.M. Twenty years of terahertz imaging. Opt. Exp. 2018, 26, 9417-9431. [CrossRef]

9. Ahmed, K.; Ahmed, F.; Roy, S.; Paul, B.K.; Aktar, M.N.; Vigneswaran, D.; Islam, M.S. Refractive index-based blood components sensing in terahertz spectrum. IEEE Sens. J. 2019, 19, 3368-3375. [CrossRef]

10. Hasanuzzaman, G.K.; Iezekiel, S.; Markos, C.; Habib, M.S. Hollow-core fiber with nested anti-resonant tubes for low-loss terahertz guidance. Opt. Commun. 2018, 426, 477-482. [CrossRef]

11. Cui, W.; Schiff-Kearn, W.A.; Zhang, E.; Couture, N.; Tani, F.; Novoa, D.; Russell, P.S.; Ménard, J.M. Broadband and tunable time-resolved THz system using argon-filled hollow-core photonic crystal fiber. APL Photonics 2018, 3, 111301. [CrossRef]

12. Yan, D.X.; Zhang, H.W.; Xu, D.G.; Shi, W.; Yan, C.; Liu, P.X.; Shi, J.; Yao, J.Q. Numerical Study of Compact Terahertz Gas Laser Based on Photonic Crystal Fiber Cavity. J. Lightwave Technol. 2016, 34, 3373-3378. [CrossRef]

13. Hossain, M.S.; Habib, M.S.; Razzak, S.M.; Markos, C.; Habib, M.S. Highly Birefringent, Low-Loss, and Near-Zero Flat Dispersion ENZ Based THz Photonic Crystal Fibers. IEEE Photon. J. 2020, 12, 7202109. [CrossRef]

14. Li, J.W.; Nallappan, K.; Guerboukha, H.; Skorobogatiy, M. 3D printed hollow core terahertz Bragg waveguides with defect layers for surface sensing applications. Opt. Exp. 2017, 25, 4126-4144. [CrossRef] 
15. Cao, Y.; Nallappan, K.; Guerboukha, H.; Gervais, T.; Skorobogatiy, M. Additive manufacturing of resonant fluidic sensors based on photonic bandgap waveguides for terahertz applications. Opt. Exp. 2019, 27, 27663-27681. [CrossRef]

16. Han, H.; Park, H.; Cho, M.; Kim, J. Terahertz pulse propagation in a plastic photonic crystal fiber. Appl. Phys. Lett. 2002, 80, 2634-2636. [CrossRef]

17. Aming, A.; Uthman, M.; Chitaree, R.; Mohammed, W.; Rahman, B.A. Design and characterization of porous-core polarization maintaining photonic crystal fiber for terahertz guidance. J. Lightwave Technol. 2016, 34, 5583-5590. [CrossRef]

18. Gallot, G.; Jamison, S.P.; McGowan, R.W.; Grischkowsky, D. Terahertz waveguides. J. Opt. Soc. Am. B 2000, 17, 851-863. [CrossRef]

19. Wang, K.; Mittleman, D.M. Metal wires for terahertz wave guiding. Nature 2004, 432, 376-379. [CrossRef]

20. Yang, J.; Yang, B.; Wang, Z.; Liu, W.W. Design of the low-loss wide bandwidth hollow-core terahertz inhibited coupling fibers. Opt. Commun. 2015, 343, 150-156. [CrossRef]

21. Shephard, J.D.; MacPherson, W.N.; Maier, R.R.; Jones, J.D.; Hand, D.P.; Mohebbi, M.; George, A.K.; Roberts, P.J.; Knight, J.C. Single-mode mid-IR guidance in a hollow-core photonic crystal fiber. Opt. Exp. 2005, 13, 7139-7144. [CrossRef]

22. Roberts, P.J.; Couny, F.; Sabert, H.; Mangan, B.J.; Williams, D.P.; Farr, L.; Mason, M.W.; Tomlinson, A.; Birks, T.A.; Knight, J.C.; et al. Ultimate low loss of hollow-core photonic crystal fibres. Opt. Exp. 2005, 13, 236-244. [CrossRef]

23. Perakis, F.; De Marco, L.; Shalit, A.; Tang, F.J.; Kann, Z.R.; Kuehne, T.D.; Torre, R.; Bonn, M.; Nagata, Y. Vibrational Spectroscopy and Dynamics of Water. Chen. Rev. 2016, 116, 7590-7607. [CrossRef] [PubMed]

24. Yiwen, E.; Jin, Q.; Tcypkin, A.; Zhang, X.-C. Terahertz wave generation from liquid water films via laser-induced breakdown. Appl. Phys. Lett. 2018, 113, 181103.

25. Yiwen, E.; Jin, Q.; Zhang, X.C. Enhancement of terahertz emission by a preformed plasma in liquid water. Appl. Phys. Lett. 2019, $115,101101$.

26. Jin, Q.; Yiwen, E.; Williams, K.; Dai, J.M.; Zhang, X.C. Observation of broadband terahertz wave generation from liquid water. Appl. Phys. Lett. 2017, 111, 071103. [CrossRef]

27. Vij, J.K.; Simpson, D.R.; Panarina, O.E. Far infrared spectroscopy of water at different temperatures: GHz to THz dielectric spectroscopy of water. J. Mol. Liq. 2004, 112, 125-135. [CrossRef]

28. Qu, H.; Skorobogatiy, M. Resonant bio- and chemical sensors using low-refractive-index-contrast liquid-core Bragg fibers. Sens. Actuators B 2012, 161, 261-268. [CrossRef]

29. Zhou, J.; Rao, X.; Liu, X.M.; Li, T.; Zhou, L.; Zheng, Y.S.; Zhu, Z. Temperature dependent optical and dielectric properties of liquid water studied by terahertz time-domain spectroscopy. Aip. Adv. 2019, 9, 035346. [CrossRef]

30. Kindt, J.T.; Schmuttenmaer, C.A. Far-Infrared Dielectric Properties of Polar Liquids Probed by Femtosecond Terahertz Pulse Spectroscopy. J. Phys. Chem. 1996, 100, 10373-10379. [CrossRef]

31. Paul, B.K.; Haque, M.A.; Ahmed, K.; Sen, S. A Novel Hexahedron Photonic Crystal Fiber in Terahertz Propagation: Design and Analysis. Photonics 2019, 6, 32. [CrossRef]

32. Islam, M.S.; Sultana, J.; Dinovitser, A.; Ng, B.W.; Abbott, D. A novel Zeonex based oligoporous-core photonic crystal fiber for polarization preserving terahertz applications. Opt. Commun. 2018, 413, 242-248. [CrossRef]

33. Paul, B.K.; Ahmed, K.; Vigneswaran, D.; Ahmed, F.; Roy, S.; Abbott, D. Quasi-Photonic Crystal Fiber-Based Spectroscopic Chemical Sensor in the Terahertz Spectrum: Design and Analysis. IEEE Sens. J. 2018, 18, 9948-9954. [CrossRef] 